\title{
Размерные и температурные зависимости ширины запрещенной зоны квантовых точек селенида кадмия во фторофосфатных стеклах
}

\author{
() Ж.О. Липатова, Е.В. Колобкова, А.Н. Бабкина, Н.В. Никоноров
}

Университет ИТМО,

197101 Санкт-Петербург, Россия

E-mail: zluka_yo@mail.ru

(Получена 18 мая 2016 г. Принята к печати 17 августа 2016 г.)

Исследованы температурные и размерные зависимости изменения ширины запрещенной зоны квантовых точек CdSe с диаметрами 2.4, 4.0 и 5.2 нм во фторофосфатных стеклах. Показано, что для квантовых точек температурный коэффициент смещения ширины запрещенной зоны $d E_{g} / d T$ отличается от значения для объемного полупроводника и строго зависит от размера наночастиц. Показано, что каждый энергетический переход квантовых точек обладает своим значением температурного коэффициента энергии $d E / d T$.

DOI: 10.21883/FTP.2017.03.44204.8330

\section{1. Введение}

Оптические и электронные свойства полупроводниковых наноструктур (квантовых точек) активно изучались в последние 20 лет [1,2]. Квантовые точки вследствие квантово-размерных эффектов имеют дискретную структуру энергетического спектра и проявляют голубой сдвиг в оптических спектрах в сравнении с объемным полупроводником. Оптические свойства квантовых точек халькогенидов кадмия активно исследовались изза их интенсивной люминесценции в видимой области спектра [3]. Стекла с квантовыми точками $\mathrm{CdSe}$ обладают нелинейной восприимчивостью третьего порядка и могут использоваться в качестве рамановских усилителей [4] и оптических излучателей [5].

Важной характеристикой материала является температурный коэффициент ширины запрещенной зоны $d E_{g} / d T$. Для полупроводниковых монокристаллов температурная зависимость ширины запрещенной зоны обусловлена в основном термическим расширением решетки и электрон-фононным взаимодействием [6-9]. Для наноразмерных структур вклад в $d E_{g} / d T$ также вносит взаимодействие межзонных состояний. В зависимости от материала и размера квантовых точек изменение температурного коэффициента $d E_{g} / d T$ происходит поразному. Известно, что для квантовых точек халькогенидов свинца температурное изменение ширины запрещенной зоны происходит по нелинейному закону [10] с температурным коэффициентом $d E_{g} / d T>0$. Для квантовых точек полупроводников группы II-VI, таких как $\mathrm{CdS}, \mathrm{CdSe}$, вследствие преобладания влияния электронфононного взаимодействия над межзонным взаимодействием температурный коэффициент $d E_{g} / d T<0$. Значения температурного коэффициента $d E_{g} / d T$ для квантовых точек халькогенидов кадмия близки к значению коэффициента $d E_{g} / d T$ для объемного полупроводника [11]. В работе [12] показано, что для квантовых точек $\mathrm{CdS}$ с размерами, близкими к радиусу экситона Бора, температурное изменение ширины запрещенной зоны происходит линейно и строго зависит от размера наночастиц.
Целью данной работы было исследование размерной и температурной зависимости ширины запрещенной зоны квантовых точек $\mathrm{CdSe}$ диаметрами 2.4, 4.0 и 5.2 нм, синтезированных во фторофосфатных стеклах. В работе также представлены экспериментальные результаты по температурным коэффициентам энергий $d E / d T$ для переходов в более высокие возбужденные состояния.

\section{2. Методика эксперимента}

Для исследования использовались фторофосфатные стекла системы $\mathrm{Na}_{2} \mathrm{O}-\mathrm{P}_{2} \mathrm{O}_{5}-\mathrm{ZnF}_{2}-\mathrm{Ga}_{2} \mathrm{O}_{3}-\mathrm{AlF}_{3}-\mathrm{NaF}$, активированные $\quad 0.05 \mathrm{CdSe}+0.5 \mathrm{ZnSe} \quad$ и $0.1 \mathrm{CdSe}+$ $+0.2 \mathrm{ZnSe}$ (концентрации указаны в мол\%). Методика синтеза фторофосфатных стекол с квантовыми точками $\mathrm{CdSe}$ проводилась аналогично [13]. Формирование квантовых точек $\mathrm{CdSe}$ происходило в результате вторичной термообработки стекол в муфельной печи с программным управлением Naberthern при температуре выше температуры стеклования. Вторичная термообработка стекол проводилась при температурах $400-420^{\circ} \mathrm{C}$ в течение 30-105 мин. Во фторофосфатных стеклах синтез квантовых точек происходит при более низких температурах и меньшем времени термообработки, чем в силикатных стеклах. Для получения минимального разброса квантовых точек по размерам синтез проводился по двухстадийной схеме: термообработка при температуре $T_{1}=300^{\circ} \mathrm{C}$ для формирования зародышей кристаллизации и последующая термообработка при температуре $T_{2}$ выше температуры стеклования до образования необходимого размера квантовых точек. Варьируя температуры $T_{1}, T_{2}$ и длительность режима вторичной термообработки, можно контролировать размеры наночастиц в стеклах. Размеры квантовых точек $\mathrm{CdSe}$ были рассчитаны с использованием спектров поглощения и данных работы [14].

Спектры оптической плотности были измерены с помощью спектрофотометра Lambda 650 (диапазон измерений $1.5-5$ эВ, время интеграции $0.5 \mathrm{c})$. Измерения 
проводились в диапазоне температур 77-295 К при помощи криостата фирмы Specac.

\section{3. Экспериментальные результаты и их обсуждение}

На рис. 1 представлены спектры оптической плотности для квантовых точек $\mathrm{CdSe}$ с размерами 2.4,
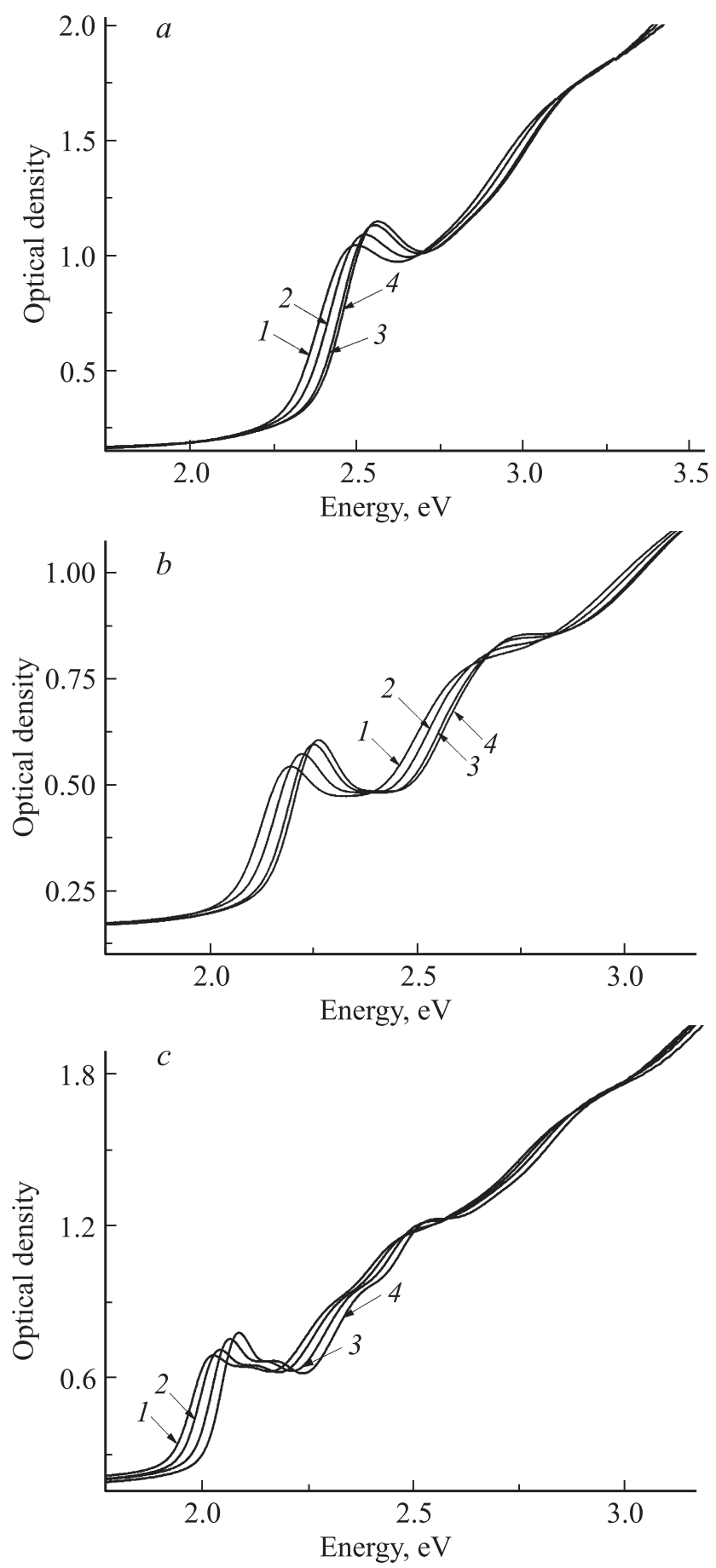

Рис. 1. Спектры поглощения квантовых точек CdSe с диаметрами $2.4(a), 4.0(b), 5.2$ нм $(c)$, измеренные при температурах 295 (1), 246 (2), 172 (3), $85 \mathrm{~K}(4)$.

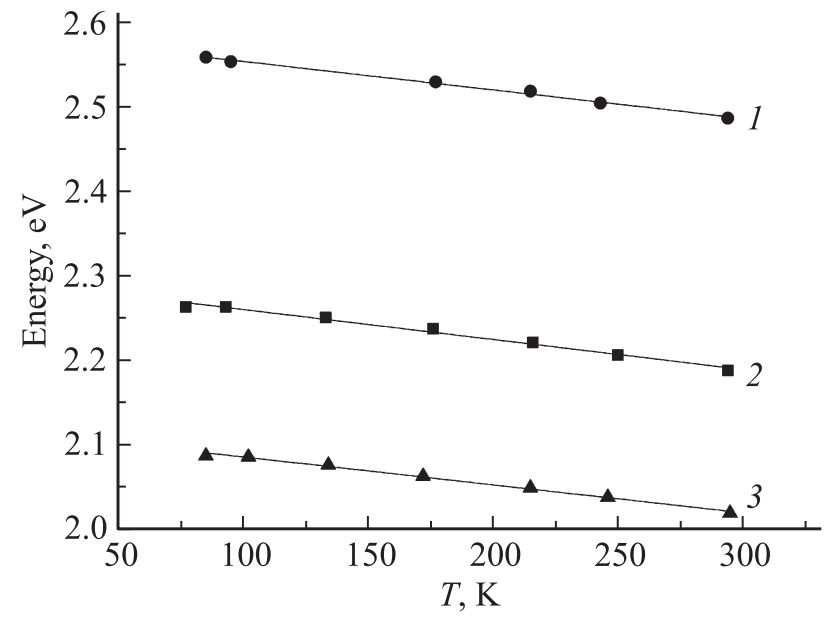

Рис. 2. Зависимости энергии первого перехода для квантовых точек CdSe с диаметрами 2.4 (1), 4.0 (2) и $5.2 \mathrm{Hм}$ (3) от температуры.

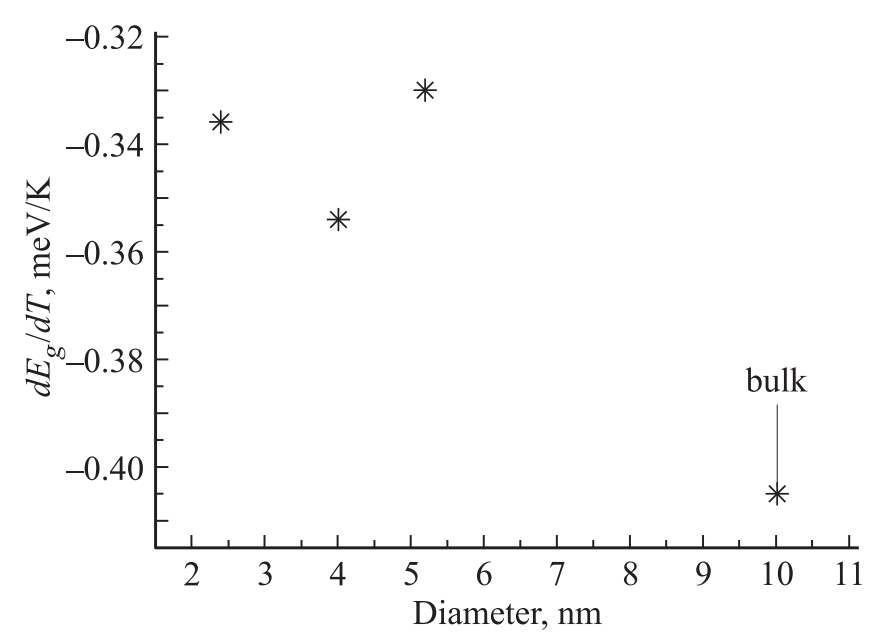

Рис. 3. Зависимость температурного коэффициента $d E_{g} / d T$ (для первого энергетического перехода) от размера квантовых точек CdSe.

4.0 и $5.2 \mathrm{Hм}$, измеренные в диапазоне температур $T=85-295 \mathrm{~K}$. Для синтезированных квантовых точек наблюдается тонкая структура спектров поглощения, на которых разрешаются несколько энергетических межзонных переходов, что обусловлено узким распределением наночастиц в матрице стекла. Уменьшение температуры с 295 до $85 \mathrm{~K}$ приводит к сдвигу полосы поглощения в область бо́льших энергий, что соответствует увеличению ширины запрещенной зоны $E_{g}$ квантовых точек CdSe. Таким образом, для всех синтезированных квантовых точек температурное изменение ширины запрещенной зоны происходит с отрицательным знаком $\left(d E_{g} / d T<0\right)$.

Зависимость сдвига первого энергетического перехода электронно-дырочной пары в спектрах поглощения от температуры приведена на рис. 2. Во всем диапазоне температур наблюдается линейная зависимость сдви- 


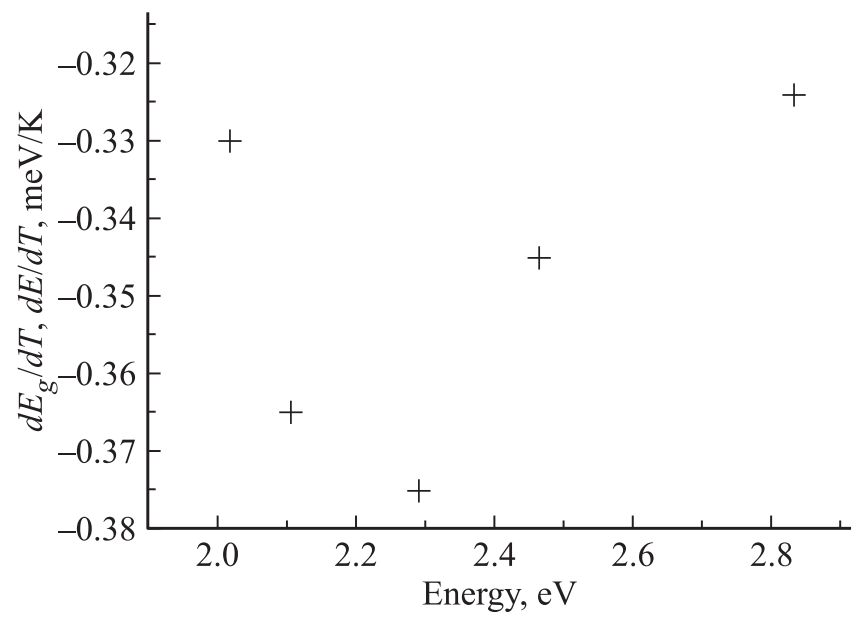

Рис. 4. Температурные коэффициенты изменения энергий переходов в разные возбужденные состояния $d E_{g} / d T, d E / d T$ для квантовых точек CdSe с диаметром 5.2 нм.

га первого максимума полосы поглощения для всех размеров квантовых точек CdSe. По данным зависимостям был рассчитан экспериментальный температурный коэффициент изменения ширины запрещенной зоны $d E_{g} / d T$ для всех размеров квантовых точек (рис. 3). Для квантовых точек $\mathrm{CdSe}$ видно отличие температурного коэффициента $d E_{g} / d T$ от такового для объемного полупроводника. Также значение коэффициента $d E_{g} / d T$ нелинейно зависит от размера квантовых точек. Так, для наночастиц с диаметрами 2.4, 4.0, $5.2 \mathrm{Hм}$ $d E_{g} / d T=-0.336,-0.354,-033$ мэВ $/ \mathrm{K}$ соответственно. Как и в работе [15], показано, что значения по модулю температурного коэффициента $d E_{g} / d T$ меньше, чем для объемного полупроводника.

Была сделана оценка значений температурного коэффициента энергий переходов $d E_{g} / d T$ в более высокие возбужденные состояния квантовых точек $\mathrm{CdSe}$ с размером 5.2 нм. На рис. 4 показано, что для каждого возбужденного состояния квантовых точек значение температурного коэффициента $d E / d T$ разное и изменяется в пределах от -0.324 до -0.375 мэВ/К.

\section{4. Заключение}

Таким образом, показано, что значения экспериментального температурного коэффициента $d E_{g} / d T$ квантовых точек CdSe отличаются от значения коэффициента для объемного полупроводника и строго зависят от размера. В зависимости от размера наночастиц температурный коэффициент смещения ширины запрещенной зоны изменяется нелинейно. Расчеты температурного коэффициента изменения энергий $d E / d T$ для переходов в более высокие возбужденные состояния показали отличия значений от $d E_{g} / d T$.

Исследование выполнено за счет гранта Российского научного фонда (проект № 14-23-00136).

\section{Список литературы}

[1] Hu Ling et al. Superlat. Microstr., 29, 67 (2001).

[2] V.I. Klimov, A.A. Mikhailovsky, S. Xu, A. Malko, J.A. Hollingsworth, C.A. Leatherdale, H.-J. Eisler, M.G. Bawendi. Science, 290, 314 (2000).

[3] G. Kaur, H. Kaur, S.K. Tripathi. AIP Conf. Proc., 1591, 420 (2014).

[4] A. Céreyon, A.M. Jurdyc, V. Martinez, E. Burov, A. Pastouret, B. Champagnon. J. Non-Cryst. Sol., 354, 3458 (2008).

[5] R. Jia, D.S. Jiang, P.H. Tan, B.Q. Sun. Appl. Phys. Lett., 79, 153 (2001).

[6] Б. Ридли. Квантовые процессы в полупроводниках (М., Мир, 1986).

[7] V. Heine, J.A. Van Vehten. Phys. Rev. B, 13, 1622 (1976).

[8] Ch. Keffer, T.M. Hayes, A. Bienenstock. Phys. Rev. Lett., 21, 1676 (1968).

[9] Ph. Allen, V. Heine. J. Phys. C.: Sol. St. Phys., 9, 2305 (1976).

[10] A. Olkhovets, R.-C. Hsu, A. Lipovskii, F.W. Wise. Phys. Rev. Lett., 81, 3539 (1998).

[11] S. Logothetidis, M. Cardona, P. Lautenschlager, M. Garriga. Phys. Rev. B, 34, 2458 (1986).

[12] T. Vossmeyer, L. Katsikas, M. Giersig, I.G. Popovic, K. Diesner, A. Chemseddine, A. Eychmüller, H. Weller. J. Phys. Chem., 98, 7665 (1994).

[13] Ж.О. Липатова, Е.В. Колобкова, В.А. Асеев. Опт. и спектр., 119, 239 (2015).

[14] D.J. Norris, M.G. Bawendi. Phys. Rev. B., 53, 16338 (1996).

[15] В.П. Кунец, Н.Р. Кулиш, Вас.П. Кунец, М.П. Лисица, Н.И. Малыш. ФТП, 36, 227 (2002).

Редактор Л.В. Шаронова

\section{Size and temperature dependence on the energy gap of cadmium selenide quantum dots in fluorine-phosphate glasses}

\section{Zh.O. Lipatova, E.V. Kolobkova, A.N. Babkina,} N.V. Nikonorov

ITMO University, 197101 St. Petersburg, Russia

Abstract We observed temperature and the size-dependent variation of the energy gap in CdSe quantum dots with diameters 2.4, 4.0 and $5.2 \mathrm{~nm}$ in fluorine-phosphate glasses. It is shown that temperature coefficient in quantum dots depends strongly on the quantum dots size and differs from the bulk value. The difference between the temperature coefficient values for energy transitions of quantum dots is shown. 